Document downloaded from:

http://hdl.handle.net/10251/105836

This paper must be cited as:

Defez Candel, E.; Tung, MM. (2018). A new type of Hermite matrix polynomial series. Quaestiones Mathematicae. 41(2):205-212. doi:10.2989/16073606.2017.1376231

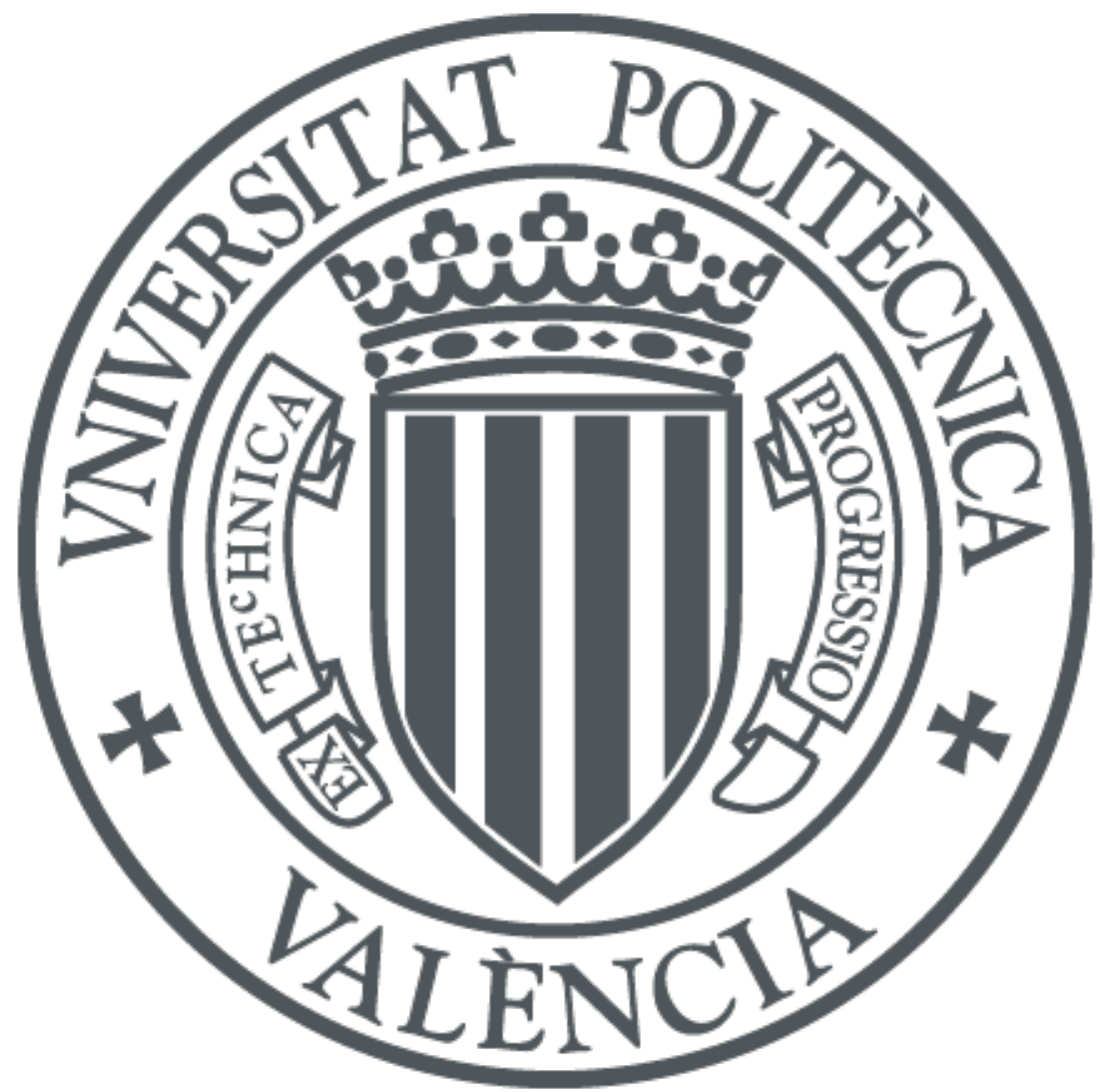

The final publication is available at

https://doi.org/10.2989/16073606.2017.1376231

Copyright Informa UK (National Inquiry Services Center)

Additional Information 


\title{
A NEW TYPE OF HERMITE MATRIX POLYNOMIAL SERIES
}

\author{
EMILIO DEFEZ* AND MICHAEL M. TUNG ${ }^{b}$
}

\begin{abstract}
Conventional Hermite polynomials emerge in a great diversity of applications in mathematical physics, engineering, and related fields. However, in physical systems with higher degrees of freedom it will be of practical interest to extend the scalar Hermite functions to their matrix analogue. This work introduces various new generating functions for Hermite matrix polynomials and examines existence and convergence of their associated series expansion by using Mehler's formula for the general matrix case. Moreover, we derive interesting new relations for even- and odd-power summation in the generatingfunction expansion containing Hermite matrix polynomials. Some new results for the scalar case are also presented.
\end{abstract}

\section{INTRODUCTION}

During the last decade, orthogonal matrix polynomials have become increasingly important for numerical computation and its analysis. In particular, Hermite matrix polynomials were introduced and studied for the first time in $[8,10]$ and subsequently received considerable attention for its application in the solutions of matrix differential equations $[2-5,12,13]$. In the scalar case, Hermite polynomials emerge in the context of quantum mechanics and optics, mathematical and nuclear physics among other areas of highly practical interest. Only recently, a new kind of series expansion involving conventional Hermite polynomials was introduced in [7] in order to describe new field states in quantum optics - yet without any rigorous proof of existence and convergence in general.

In this article, we present an entirely new class of series expansion for Hermite matrix polynomials, which also includes the scalar polynomial expansion of [7] as a particular case. Besides, we will provide an accurate analysis of convergence.

To start with, in Section 2, we define the new Hermite matrix polynomial series. Section 3 illustrates the reduction to the scalar case, given in [7]. Throughout this work, we denote by $\mathbb{C}^{r \times r}$ the set of all the complex square matrices of size $r$. Furthermore, by $\Theta$ and $I$ we denote the zero and the identity matrix, respectively. If $f(z), g(z)$ are holomorphic functions on an open set $\Omega \subset \mathbb{C}$, and if the eigenvalues $\sigma(A) \subset \Omega$, then $f(A), g(A)$ represent the images of functions $f$ and $g$ acting on the matrix $A$ such that $f(A) g(A)=g(A) f(A)$, see [6, p.558]. If $\operatorname{Re}(z)>0$ for every eigenvalue $z \in \sigma(A)$, we say that matrix $A$ is positive stable, In this case, we use $\sqrt{A}=A^{1 / 2}=\exp (\log (A) / 2)$ for the image of the function $z^{1 / 2}=\exp (\log (z) / 2)$

2000 Mathematics Subject Classification. 33C45, 33C47, 15A16.

Key words and phrases. Hermite matrix polynomials, Hermite polynomials, generating functions.

The authors thank the Spanish Ministerio de Economía y Competitividad and the European Regional Development Fund (ERDF) for financial support under grant TIN2014-59294-P. 
acting on the matrix $A$. Note that $\log (z)$ as usual denotes the principal branch of the complex logarithm.

A matrix polynomial of degree $n$ is an expression of the form $P_{n}(t)=A_{n} t^{n}+$ $A_{n-1} t^{n-1}+\cdots+A_{1} t+A_{0}$, where $t$ is a real variable and $A_{j} \in \mathbb{C}^{r \times r}$ for $0 \leq j \leq n$. The usual 2-norm of a quadratic matrix $A$ is denoted by $\|A\|_{2}$.

\section{The New Hermite MAtrix POlynomial SERIES}

For any positive stable matrix $A \in \mathbb{C}^{r \times r}$, the associated Hermite matrix polynomials [8] are defined by the following three-term recurrence for any non-zero positive integer $m \in \mathbb{N}$ :

$H_{-1}(x, A)=\Theta, H_{0}(x, A)=I, H_{m}(x, A)=x \sqrt{2 A} H_{m-1}(x, A)-2(m-1) H_{m-2}(x, A)$.

This definition fulfills for any $n \in \mathbb{N}_{0}$ (positive integers including zero) the relations

$$
H_{2 n+1}(0, A)=\Theta, \quad H_{2 n}(0, A)=(-1)^{n} \frac{(2 n) !}{n !} I .
$$

For the following derivations we need to use two well-known results. The first result was demonstrated in [1] and establishes the following upper bound:

$$
\begin{aligned}
& \left\|H_{2 n}(x, A)\right\|_{2} \leq g_{n}(x), \quad\left\|H_{2 n+1}(x, A)\right\|_{2} \leq|x|\left\|\left(\frac{A}{2}\right)^{-1 / 2}\right\|_{2} \frac{2 g_{n}(x)}{n+1}, \\
& g_{n}(x)=2^{2 n} \frac{(2 n+1) !}{n !} \exp \left(\frac{5}{2}\|A\|_{2} x^{2}\right), \quad n \in \mathbb{N}_{0} .
\end{aligned}
$$

The second result was demonstrated in [9] and is Mehler's formula in the matrix case:

$$
\sum_{n \geq 0} \frac{H_{n}(x, A) H_{n}(y, A)}{2^{n} n !} t^{n}=\left(1-t^{2}\right)^{-\frac{1}{2}} \exp \left(\frac{2 x y t-\left(x^{2}+y^{2}\right) t^{2}}{2\left(1-t^{2}\right)} A\right), x, y \in \mathbb{R},|t|<1 .
$$

Now we are in the position to prove the following theorem:

Theorem 2.1. Let $A \in \mathbb{C}^{r \times r}$ be a positive stable matrix and $x \in \mathbb{R}$. Then for $|t|<1 / 16$

$$
\mathcal{A}(s ; x, t, A):=\sum_{n \geq 0} \frac{H_{2 n+s}(x, A)}{n !} t^{n}=\frac{H_{s}\left(\frac{x}{\sqrt{1+4 t}}, A\right)}{(\sqrt{1+4 t})^{s+1}} \exp \left(\frac{2 t x^{2}}{1+4 t} A\right), s \in \mathbb{N}_{0} .
$$

Proof 2.1. First we will prove that the matrix series $\mathcal{A}(s ; x, t, A)$ is convergent for any fixed integer $s \in \mathbb{N}_{0}$. Taking into account (2.3), being $s=2 l$ an even number, one obtains

$$
\left\|\frac{H_{2 n+s}(x, A)}{n !} t^{n}\right\|_{2}=\left\|\frac{H_{2 n+2 l}(x, A)}{n !} t^{n}\right\|_{2} \leq \frac{g_{n+l}(x)|t|^{n}}{n !} .
$$

Since $\sum_{n \geq 0} \frac{g_{n+l}(x)|t|^{n}}{n !}$ is convergent for $|t|<1 / 16$, the matrix series $\mathcal{A}(2 l ; x, t, A)$ is convergent in any compact real interval. On other hand, if $s=2 l+1$ is an odd 
number, then

$$
\left\|\frac{H_{2 n+s}(x, A)}{n !} t^{n}\right\|_{2}=\left\|\frac{H_{2(n+l)+1}(x, A)}{n !} t^{n}\right\|_{2} \leq|x|\left\|(A / 2)^{-1 / 2}\right\|_{2} \frac{2 g_{n+l}(x)|t|^{n}}{(n+l+1) n !} .
$$

Since $\sum_{n \geq 0} \frac{|x|\left\|(A / 2)^{-1 / 2}\right\|_{2} 2 g_{n+l}(x)|t|^{n}}{(n+l+1) n !}$ is convergent for $|t|<1 / 16$, the matrix series $\mathcal{A}(2 l+1 ; x, t, A)$ is convergent in any compact real interval. Thus, the series $\mathcal{A}(s ; x, t, A)$ is convergent for all fixed integers $s \in \mathbb{N}_{0}$, what was to be shown.

For proving formula (2.5), we will use the induction method. We put $y=0$ in formula (2.4) and use relations (2.2). Thus, Mehler's formula (2.4) reduces to (see [1] for details):

$$
\sum_{n \geq 0} \frac{(-1)^{n} H_{2 n}(x, A)}{2^{2 n} n !} t^{2 n}=\left(1-t^{2}\right)^{-\frac{1}{2}} \exp \left(\frac{-x^{2} t^{2}}{2\left(1-t^{2}\right)} A\right), x \in \mathbb{R},|t|<1 / 2 .
$$

Taking $u=-t^{2} / 4$, we can rewrite (2.6) in the form

$$
\sum_{n \geq 0} \frac{H_{2 n}(x, A)}{n !} u^{n}=(1+4 u)^{-\frac{1}{2}} \exp \left(\frac{2 u x^{2}}{1+4 u} A\right), \quad x \in \mathbb{R}, \quad|u|<1 / 16 .
$$

Observe that $(2.7)$ is exactly $\mathcal{A}(0 ; x, u, A)$. Thus, formula $(2.5)$ is true for $s=0$.

Next, we proceed to prove that formula (2.5) is also true for $s=1$. After using the three-term recurrence (2.1) for $m=2 n+1$, multiplying each term by $t^{n} / n$ ! with $|t|<1 / 16$, and finally applying the sum from $n=1$ to infinity, one arrives at

$$
\sum_{n \geq 1} \frac{H_{2 n+1}(x, A)}{n !} t^{n}=x \sqrt{2 A} \sum_{n \geq 1} \frac{H_{2 n}(x, A)}{n !} t^{n}-4 \sum_{n \geq 1} \frac{n H_{2 n-1}(x, A)}{n !} t^{n} .
$$

To start summation at $n=0$, we rewrite $(2.8)$ in the form

$$
(1+4 t) \sum_{n \geq 0} \frac{H_{2 n+1}(x, A)}{n !} t^{n}=H_{1}(x, A)-x \sqrt{2 A} H_{0}(x, A)+x \sqrt{2 A} \sum_{n \geq 0} \frac{H_{2 n}(x, A)}{n !} t^{n} .
$$

Applying (2.1), we conclude that $H_{1}(x, A)=x \sqrt{2 A}, H_{0}(x, A)=I$. Now, using (2.7), we may simplify (2.9) to the form:

$$
\mathcal{A}(1 ; x, t, A)=\frac{x \sqrt{2 A}}{(\sqrt{1+4 t})^{3}} \exp \left(\frac{2 t x^{2}}{1+4 t} A\right)=\frac{H_{1}\left(\frac{x}{\sqrt{1+4 t}}, A\right)}{(\sqrt{1+4 t})^{2}} \exp \left(\frac{2 t x^{2}}{1+4 t} A\right),
$$

and formula (2.5) is also true for $s=1$.

Up to this point we only have shown that the inductive statement $\mathcal{A}(s ; x, u, A)$ holds for $s=0,1$. Next, we proceed with the inductive step, showing that if $\mathcal{A}(l ; x, u, A)$ holds for $0 \leq l \leq s-1$, then $\mathcal{A}(s ; x, u, A)$ is also true.

Again, we use the recurrence relation (2.1) for $m=2 n+s$ to obtain

$$
H_{2 n+s}(x, A)=x \sqrt{2 A} H_{2 n+s-1}(x, A)-2(s-1) H_{2 n+s-2}(x, A)-4 n H_{2 n+s-2}(x, A) .
$$


Multiplying (2.11) by $t^{n} / n$ ! with $|t|<1 / 16$, and applying the sum from $n=1$ to infinity, we deduce

$$
\begin{aligned}
\sum_{n \geq 1} \frac{H_{2 n+s}(x, A)}{n !} t^{n}= & \sqrt{2 A} \sum_{n \geq 1} \frac{H_{2 n+s-1}(x, A)}{n !} t^{n}-2(s-1) \sum_{n \geq 1} \frac{H_{2 n+s-2}(x, A)}{n !} t^{n} \\
& -4 \sum_{n \geq 1} \frac{n H_{2 n+s-2}(x, A)}{n !} t^{n}
\end{aligned}
$$

We can rewrite $(2.12)$ in the form

$$
\begin{aligned}
\mathcal{A}(s ; x, t, A)= & H_{s}(x, A)-x \sqrt{2 A} H_{s-1}(x, A)-2(s-1) H_{s-2}(x, A) \\
& +x \sqrt{2 A} \mathcal{A}(s-1 ; x, t, A)-2(s-1) \mathcal{A}(s-2 ; x, t, A)-4 t \mathcal{A}(s ; x, t, A) .
\end{aligned}
$$

The first three terms of equation (2.13) reduce to $H_{s}(x, A)-x \sqrt{2 A} H_{s-1}(x, A)-$ $2(s-1) H_{s-2}(x, A)=\Theta$ by using recurrence $(2.1)$ for $m=s$. Next, we can simplify expression (2.13) to

$$
(1+4 t) \mathcal{A}(s ; x, u, A)=x \sqrt{2 A} \mathcal{A}(s-1 ; x, u, A)-2(s-1) \mathcal{A}(s-2 ; x, u, A) .
$$

Using the induction hypothesis, we have

$$
\left.\begin{array}{l}
\mathcal{A}(s-1 ; x, u, A)=\frac{H_{s-1}\left(\frac{x}{\sqrt{1+4 t}}, A\right)}{(\sqrt{1+4 t})^{s}} \exp \left(\frac{2 t x^{2}}{1+4 t} A\right) \\
\mathcal{A}(s-2 ; x, u, A)=\frac{H_{s-2}\left(\frac{x}{\sqrt{1+4 t}}, A\right)}{(\sqrt{1+4 t})^{s-1}} \exp \left(\frac{2 t x^{2}}{1+4 t} A\right)
\end{array}\right\} .
$$

Substituting (2.15) in (2.14), one finally arrives at

$$
\begin{aligned}
(1+4 t) \mathcal{A}(s ; x, u, A)=x \sqrt{2 A}\left(\frac{H_{s-1}\left(\frac{x}{\sqrt{1+4 t}}, A\right)}{(\sqrt{1+4 t})^{s}} \exp \left(\frac{2 t x^{2}}{1+4 t} A\right)\right) \\
-2(s-1)\left(\frac{H_{s-2}\left(\frac{x}{\sqrt{1+4 t}}, A\right)}{(\sqrt{1+4 t})^{s-1}} \exp \left(\frac{2 t x^{2}}{1+4 t} A\right)\right) \\
=\frac{\exp \left(\frac{2 t x^{2}}{1+4 t} A\right)}{(\sqrt{1+4 t})^{s-1}}\left[\frac{x \sqrt{2 A}}{(\sqrt{1+4 t})} H_{s-1}\left(\frac{x}{\sqrt{1+4 t}}, A\right)-2(s-1) H_{s-2}\left(\frac{x}{\sqrt{1+4 t}}, A\right)\right] \\
=\frac{H_{s}\left(\frac{x}{\sqrt{1+4 t}}, A\right)}{(\sqrt{1+4 t})^{s-1}} \exp \left(\frac{2 t x^{2}}{1+4 t} A\right)
\end{aligned}
$$

which completes this proof.

Remark 2.1. Working with the series $\mathcal{A}(s ; x, t, A)$ and its new results established so far, we may continue to derive additional, previously unknown relations and properties of the Hermite matrix series, altogether absent from the literature on special functions. 
For example, considering the combinations $\mathcal{A}(s ; x, t, A)+\mathcal{A}(s ; x,-t, A)$ and also $\mathcal{A}(s ; x, t, A)-\mathcal{A}(s ; x,-t, A)$, for $s \in \mathbb{N}_{0}, x \in \mathbb{R},|t|<1 / 16$ it immediately follows that

$$
\begin{aligned}
\sum_{n \geq 0} \frac{H_{4 n+s}(x, A)}{(2 n) !} t^{2 n} & =\frac{H_{s}\left(\frac{x}{\sqrt{1+4 t}}, A\right)}{2(\sqrt{1+4 t})^{s+1}} \exp \left(\frac{2 t x^{2}}{1+4 t} A\right) \\
& +\frac{H_{s}\left(\frac{x}{\sqrt{1-4 t}}, A\right)}{2(\sqrt{1-4 t})^{s+1}} \exp \left(\frac{-2 t x^{2}}{1-4 t} A\right), \\
\sum_{n \geq 0} \frac{H_{4 n+s+2}(x, A)}{(2 n+1) !} t^{2 n+1}= & \frac{H_{s}\left(\frac{x}{\sqrt{1+4 t}}, A\right)}{2(\sqrt{1+4 t})^{s+1}} \exp \left(\frac{2 t x^{2}}{1+4 t} A\right) \\
& -\frac{H_{s}\left(\frac{x}{\sqrt{1-4 t}}, A\right)}{2(\sqrt{1-4 t})^{s+1}} \exp \left(\frac{-2 t x^{2}}{1-4 t} A\right) .
\end{aligned}
$$

Considering now the combinations $\mathcal{A}(s+1 ; x, t, A)+\mathcal{A}(s+1 ; x,-t, A)$ and also $\mathcal{A}(s+1 ; x, t, A)-\mathcal{A}(s+1 ; x,-t, A)$, for $s \in \mathbb{N}_{0}, x \in \mathbb{R}$, and $|t|<1 / 16$, it immediately follows that

$$
\begin{aligned}
\sum_{n \geq 0} \frac{H_{4 n+s+1}(x, A)}{(2 n) !} t^{2 n} & =\frac{H_{s+1}\left(\frac{x}{\sqrt{1+4 t}}, A\right)}{2(\sqrt{1+4 t})^{s+2}} \exp \left(\frac{2 t x^{2}}{1+4 t} A\right) \\
& +\frac{H_{s+1}\left(\frac{x}{\sqrt{1-4 t}}, A\right)}{2(\sqrt{1-4 t})^{s+2}} \exp \left(\frac{-2 t x^{2}}{1-4 t} A\right) \\
\sum_{n \geq 0} \frac{H_{4 n+s+3}(x, A)}{(2 n+1) !} t^{2 n+1} & =\frac{H_{s+1}\left(\frac{x}{\sqrt{1+4 t}}, A\right)}{2(\sqrt{1+4 t})^{s+2}} \exp \left(\frac{2 t x^{2}}{1+4 t} A\right) \\
& -\frac{H_{s+1}\left(\frac{x}{\sqrt{1-4 t}}, A\right)}{2(\sqrt{1-4 t})^{s+2}} \exp \left(\frac{-2 t x^{2}}{1-4 t} A\right)
\end{aligned}
$$

\section{The scalar Hermite polynomial series ReVisited}

Clearly, all newly proposed relations for the Hermite matrix polynomials also subsume the conventional scalar case. This puts us into the position to easily recover the scalar formula (3.1) derived by the authors of [7] within the context of quantum mechanics via what they call the quantum mechanical operator-Hermite polynomial method. 
From a purely mathematical standpoint, we can deduce the following result from Theorem 2.1:

Corollary 3.1. Let $\left\{H_{n}(x)\right\}_{n \geq 0}$ be the usual sequence of scalar Hermite polynomials. Then for $s \in \mathbb{N}_{0}, x \in \mathbb{R},|t|<1 / 4$ :

$$
\begin{aligned}
& \sum_{n \geq 0} \frac{H_{2 n+s}(x)}{n !} t^{n}=\frac{H_{s}\left(\frac{x}{\sqrt{1+4 t}}\right)}{(\sqrt{1+4 t})^{s+1}} \exp \left(\frac{4 t x^{2}}{1+4 t}\right), \\
& \sum_{n \geq 0} \frac{H_{4 n+s}(x)}{(2 n) !} t^{2 n}=\frac{H_{s}\left(\frac{x}{\sqrt{1+4 t}}\right)}{2(\sqrt{1+4 t})^{s+1}} \exp \left(\frac{4 t x^{2}}{1+4 t}\right)+\frac{H_{s}\left(\frac{x}{\sqrt{1-4 t}}\right)}{2(\sqrt{1-4 t})^{s+1}} \exp \left(\frac{-4 t x^{2}}{1-4 t}\right), \\
& \sum_{n \geq 0} \frac{H_{4 n+s+2}(x)}{(2 n+1) !} t^{2 n+1}=\frac{H_{s}\left(\frac{x}{\sqrt{1+4 t}}\right)}{2(\sqrt{1+4 t})^{s+1}} \exp \left(\frac{4 t x^{2}}{1+4 t}\right)-\frac{H_{s}\left(\frac{x}{\sqrt{1-4 t}}\right)}{2(\sqrt{1-4 t})^{s+1}} \exp \left(\frac{-4 t x^{2}}{1-4 t}\right), \\
& \sum_{n \geq 0} \frac{H_{4 n+s+1}(x)}{(2 n) !} t^{2 n}=\frac{H_{s+1}\left(\frac{x}{\sqrt{1+4 t}}\right)}{2(\sqrt{1+4 t})^{s+2}} \exp \left(\frac{4 t x^{2}}{1+4 t}\right)+\frac{H_{s+1}\left(\frac{x}{\sqrt{1-4 t}}\right)}{2(\sqrt{1-4 t})^{s+2}} \exp \left(\frac{-4 t x^{2}}{1-4 t}\right) \\
& \sum_{n \geq 0} \frac{H_{4 n+s+3}(x)}{(2 n+1) !} t^{2 n+1}=\frac{H_{s+1}\left(\frac{x}{\sqrt{1+4 t}}\right)}{2(\sqrt{1+4 t})^{s+2}} \exp \left(\frac{4 t x^{2}}{1+4 t}\right)-\frac{H_{s+1}\left(\frac{x}{\sqrt{1-4 t}}\right)}{2(\sqrt{1-4 t})^{s+2}} \exp \left(\frac{-4 t x^{2}}{1-4 t}\right) .
\end{aligned}
$$

Proof 3.1. It is well known that when $A=2$ with matrix dimension $r=1$, the Hermite matrix polynomials $H_{n}(x, A)$ reduce to their scalar counterparts, the usual Hermite polynomials $H_{n}(x)$. After substituting these values into formulas (2.5), (2.16) and (2.17), we recover formulas (3.1)-(3.2).

What remains is to prove the change in the interval of convergence, having $|t|<1 / 4$ instead of $|t|<1 / 16$. For the scalar case, we use the following bound derived by Cramer [11],

$$
\left|H_{n}(x)\right| \leq K 2^{n / 2} \sqrt{n !} e^{x^{2} / 2}, \quad K=1.086435,
$$

to finally obtain the interval of convergence $|t|<1 / 4$.

\section{REFERENCES}

[1] E. Defez, A. Hervás, L. Jódar and A. Law. Bounding Hermite matrix polynomials. Math. Comput. Model. 40 (2004)(1), 117-125.

[2] E. Defez And L. JódAR. Some applications of the Hermite matrix polynomials series expansions. J. Comput. Appl. Math. 99 (1998)(1), 105-117.

[3] E. Defez, J. Sastre, J. Ibáñez and J. Peinado. Solving engineering models using hyperbolic matrix functions. Appl. Math. Model. 40 (2016)(4), 2837-2844.

[4] E. Defez, J. Sastre, J. Ibáñez and P. Ruiz. Computing matrix functions arising in engineering models with orthogonal matrix polynomials. Math. Comput. Model. 57 (2013)(7), $1738-1743$.

[5] E. Defez, J. Sastre, J. Ibáñez And P. A. Ruiz. Computing matrix functions solving coupled differential models. Math. Comput. Model. 50 (2009)(5), 831-839. 
[6] N. Dunford and J. T. Schwartz. Linear Operators, Part I, John Wiley \& Sons, Inc., New York, 1958

[7] F. Hong-Yi and Z. De-Hui. New generating function formulae of even-and odd-Hermite polynomials obtained and applied in the context of quantum optics. Chinese Phys. B 23 (2014)(6), 060301.

[8] J. JóDAR AND R. COMPANY. Hermite matrix polynomials and second order matrix differential equations. Approx. Theor. Appl. 12 (1996)(2), 20-30.

[9] L. Jódar AND E. Defez. A matrix formula for the generating function of the product of Hermite matrix polynomials. In International Workshop on Orthogonal Polynomials in Mathematical Physics, SIAM, Madrid, Spain, 1996, pp. 93-101.

[10] L. Jódar And E. Defez. On Hermite matrix polynomials and Hermite matrix functions. Approx. Theor. Appl. 14 (1998)(1), 36-48.

[11] G. Sansone. Orthogonal Functions, Interscience Publishers, New York, 1959.

[12] J. Sastre, J. Ibáñez, E. Defeez and P. Ruiz. Efficient orthogonal matrix polynomial based method for computing matrix exponential. Appl. Math. Comput. 217 (2011)(14), 6451-6463.

[13] J. Sastre, J. Ibáñez, P. Ruiz and E. Defez. Efficient computation of the matrix cosine. Appl. Math. Comput. 219 (2013)(14), 7575-7585.

*Instituto de Matemática Multidisciplinar, Universitat Politècnica de València, Camino de Vera, s/n, 46022 Valencia, Spain

E-mail address: edefez@imm.upv.es

binstituto de Matemática Multidisciplinar, Universitat Politècnica de València, Camino de Vera, s/n, 46022 Valencia, Spain

E-mail address: mtung@imm.upv.es 